\title{
Effect of propofol on local field potential in rat prefrontal cortex during working memory task
}

\author{
Xinyu Xu ${ }^{1}$, Guolin Wang ${ }^{2}$, Wenqian Zhai ${ }^{2}$, Wenwen Bai ${ }^{1}$, Tiaotiao Liu ${ }^{1}$, Xin Tian ${ }^{{ }^{*}}$ \\ ${ }^{1}$ School of Biomedical Engineering, Tianjin Medical University, Tianjin, China \\ ${ }^{2}$ Department of Anesthesiology, Tianjin General Hospital, Tianjin Medical University, Tianjin, China \\ Email: ${ }^{*}$ tianx@tijmu.edu.cn
}

Received 10 May 2012; revised 11 June 2012; accepted 25 June 2012

\begin{abstract}
Propofol may produce memory impairment during anesthesia procedure. Local field potentials (LFPs) are used with increasing frequency in recent years to link neural activity to perception and cognition. In this study, effect of propofol on LFPs in rat's prefrontal cortex during working memory task was evaluated. Young (approximately 3 months) male Sprague-Dawley rats were divided into two group: propofol rats and control rats. Propofol rats received propofol at $0.9 \mathrm{mg} \cdot \mathrm{kg}^{-1} \cdot \mathrm{min}^{-1}$ intravenously for $2 \mathrm{~h}$. After $12 \mathrm{~h}$, LFPs of all rats were measured simultaneously from multiple electrodes placed in prefrontal cortex while rats were performing a working memory task in $\mathrm{Y}$ maze. LFPs instantaneous phase were obtained by applying Hilbert transform, and cross-correlation coherence of LFPs was calculated. The results indicate that propofol decreased the correct rate and crosscorrelation coherence of LFPs on the first two days $(p<0.05)$, but had no effect on the third day $(p>0.05)$. Our results suggest that propofol can impair crosscorrelation coherence of LFPs in the first two days, but not long time.
\end{abstract}

Keywords: Propofol; Working Memory; Local Field Potential; Rat

\section{INTRODUCTION}

\subsection{Propofol}

Propofol (2,6-diisopropylphenol) is an intravenous anesthetic that has been gaining use for induction and maintenance of anesthesia in clinical practice during the last 10 years. With the use of propofol there are some research reports about memory dysfunction caused by propofol anesthesia especially in elderly patients [1-3]. It remains unclear, however, whether these cognitive or memory changes are due to the effects of propofol.

"Corresponding author.

\subsection{Working Memory}

Working memory is the ability to actively hold information in the mind needed to do complex tasks such as reasoning, comprehension and learning [4]. Working memory tasks are those that require the goal-oriented active monitoring or manipulation of information or behaviors in the face of interfering processes and distractions. The cognitive processes involved include the executive and attention control of short-term memory which provide for the interim integration, processing, disposal, and retrieval of information.

\subsection{Memory Mechanism of Neuron Cell Assemble}

Hebb's cell assembly theory states that "Neurons do not individually represent memories but a large group of simultaneously active neurons account for any concept or idea in the human brain. Central to his theory is the idea of cell assemblies, which are formed as a result of the proposed learning process" [5]. So we research effects of propofol on working memory through the electric activities of neuron assembles.

\subsection{Prefrontal Cortex}

Behavioral studies have shown that damage in the prefrontal cortex (PFC) impairs performance in working memory tasks across various mammalian species, indicating that the PFC plays an essential role in working memory [6]. Physiological studies suggest that working memory is mediated by continuous activities of PFC neurons $[7,8]$. These studies have led to the current prevailing view that persistent neuronal activity in the PFC plays an essential role in mediating working memory [9-11].

\subsection{Local Field Potential}

The local field potential (LFP) is composed of low-frequency extracellular voltage fluctuations that are thought to reflect synaptic potentials and other slow electrical 
signals such as spike afterpotentials and voltage-dependent membrane oscillations [12,13]. Neurophysiologists have used the LFP with increasing frequency in recent years to link neural activity to perception and cognition, including sensory stimuli coding, perceptual binding, attention, and working memory [14-22].

In the present study, we aimed to determine effects of propofol anesthesia on working memory. To test this, the propofol infusion continued at a rate of $0.9 \mathrm{mg} \cdot \mathrm{kg}^{-1} \cdot \mathrm{min}^{-1}$ via a tail vein catheter for two hours. And then we simultaneously recorded LFPs from the PFC during working memory test of adult rats, using chronically implanted multielectrode arrays [23]. At last we tested the effect of propofol in rats in both LFPs and behavior tests.

\section{MATERIALS AND METHODS}

\subsection{Subjects and Housing Conditions}

A total of 20 male Sprague-Dawley rats (approximately 3 months), purchased from Institute of Radiation Medicine Chinese Academy of Medical Sciences, were housed in standard shoebox cages, had free access to drinking water, and were kept under a 12-h light-dark cycle. The rats' care and surgical procedures were in accordance with the National Institutes of Health Guide for the Care and Use of Laboratory Animals (1997) and with the Tianjin Medical University guidelines for the use and care of laboratory animals in research.

\subsection{Y-Maze Training Procedure}

Rats were food deprived to $85 \%$ of their free-feeding body weight. Behavioral training was conducted in a Ymaze (Figure 1). Both the start arm (75 cm long) and the two arms forming the $\mathrm{Y}$ (both $75 \mathrm{~cm}$ long and diverging at a $120^{\circ}$ angle from the stem arm) were $5 \mathrm{~cm}$ in width. Each animal was given 3 days of 30 min pre-training in order to train them to run reliably down the stem of the maze and to find peanut tablets in the food wells in both arms.

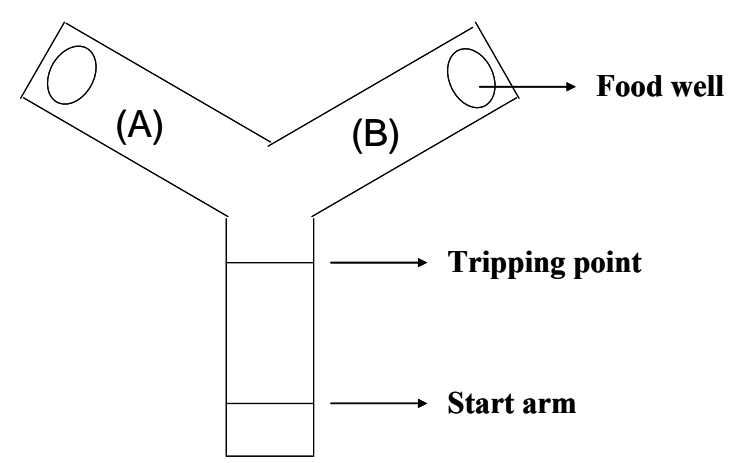

Figure 1. Y type maze used for rats behavior training and testing.
Following pre-training, the training began. At the start of training, which consisted of two stages, two peanut tablets were placed in each food well and a translucent plastic block was placed at the neck of one arm. As a consequence, on each "sample run" the rat would enter the open arm where it was allowed to eat the food at the end of the arm. The rat was then picked up and confined at the start stem for a delay of $10 \mathrm{~s}$, during which the translucent plastic block was removed. The door at the start arm was then opened and the animal allowed a free choice between the two arms of the Y-maze. On this "choice run", the animal was considered "right" if it had entered the arm not previously entered on the "sample run" and would then be allowed to eat the food reward before being returned to the start stem. If the rat returned to the arm visited on the "sample run", it was recorded "incorrect" and confined to that arm for $5 \mathrm{~s}$ before being returned to the breed box. The training procedure was not ended until each rat's the correct rate was over $80 \%$.

\subsection{Surgical Procedure}

The surgical procedure has already been described in detail [24]. All SD rats were implanted with custom-built 16-wire-S-isonel-coated tungsten microwire electrode arrays $(30 \mu \mathrm{m}$ diameter wires with $(600-800 \mathrm{k} \Omega)$ impedance, arranged in a $2 \times 8$ grid with $200 \mu \mathrm{m}$ spacing between wires) in the PFC under propofol $\left(0.5 \mathrm{mg} \cdot \mathrm{kg}^{-1} \cdot \mathrm{min}^{-1}\right)$ anesthesia. The following coordinates (in millimeters) relative to Bregma were used to center the arrays: PFCx (2.5 - $4.5 \mathrm{~mm}$ anterior to bragma and $0.2-1.0 \mathrm{~mm}$ lateral to midline, 2.5 - $3 \mathrm{~mm}$ deep from cortical surface).

\subsection{LFPs Recording and Processing}

Rats were allowed 7 days to recover from the surgery. We separated all the rats into two groups. Ten rats were injected propofol at a rate of $0.9 \mathrm{mg} \cdot \mathrm{kg}^{-1} \cdot \mathrm{min}^{-1}$ via a tail vein catheter for two hours; and the other ten rats, injected no propofol, were as the control.

After 12 hours, all the propofol group rats have recovered from anesthesia and moved as freely as the control ones. Two group rats were placed into the Y-maze to do the working memory test one by one. The test procedure was the same as the training procedure. Every rat completed continuous 10 tests in the morning and continuous 10 in the afternoon, with inter-test interval $2 \mathrm{~min}$. The rats received 20 tests a day until the two rat groups' correct rates had no differences.

At the same time, LFPs were recorded from the multielectrodes arrays and continuously sampled at $2 \mathrm{kHz}$ by using Cerebus 128 Channel Data Acquisition System (Cyberkinetics, Inc., US). The LFP signal was band-pass filtered between $0.3-500 \mathrm{~Hz}$ to remove low-frequency direct current fluctuations and reduce high-frequency 
noise. Then $50 \mathrm{~Hz}$ power line noise was removed by applying a band pass-filter to the original data, and subtracting the resulting filtered signal from the original data.

Estimate instantaneous phase by Hilbert transform: For an arbitrary signal $x(t)$, the analytic signal is a complex function of time defined as Eq.1.

$$
z(t)=x(t)+j x(t)=E(t) e^{j \psi(t)}
$$

where the function $x(t)$ is the Hilbert transform of $x(t), \psi(t)$ instantaneous phase.

Calculate cross-correlation coherence: if $\psi_{x}(t)$ and $\psi_{y}(t)$ are the instantaneous phase corresponded to two sequences of LFPs, the cross-correlation value is calculated as Eq.2.

$$
\rho_{x y}(\tau)=\frac{\int_{-\infty}^{\infty} \psi_{x}(t) \psi_{y}(t-\tau) \mathrm{d} t}{\left[\int_{-\infty}^{\infty} \psi_{x}^{2}(t) \mathrm{d} t \int_{-\infty}^{\infty} \psi_{y}^{2}(t) \mathrm{d} t\right]^{1 / 2}}
$$

where $\tau$ is time delay, $\rho_{x y}(\tau)$ is cross-correlation value $[25,26]$.

Cross-correlation coherence is used to estimate the degree of synchronization between two signals in the frequency domain. Cross-correlation values are normalized in the $[0,1]$ interval.

The zero coherence means completely desynchronized, while unit coherence means in perfect synchrony between the two signals.

In this study, we compute the cross-correlation coherences of 16-channel LFPs in $4 \mathrm{~s}$ during rats' working memory test. They are obtained by $50 \mathrm{~ms}$ sliding window and $25 \%$ overlapping. As the second channel has medium average neural firing rate of neurons, it is used as the reference channel to do the coherence with other channels.

\subsection{Data Analyses}

Data from working memory test and cross-correlation coefficient between the two LFPs were tested using repeated measures ANOVA. A limit for significance was $\mathrm{P}$ $<0.05$. All data are given as means $\pm \mathrm{SEM}$.

\section{RESULTS}

Results are based on data from 20 rats and 400 experimental sessions.

\subsection{Working Memory Y-Maze Test}

For the 3 days of test, where each test was separated by an inter-trial interval of $2 \mathrm{~min}$ and comprised a sample and choice run, an analysis carried out on the total correct choices revealed a significant group difference $[\mathrm{F}(1,18)$
$=14.7, \mathrm{P}<0.01]$ in the first day and $[\mathrm{F}(1,18)=7.3, \mathrm{P}<$ $0.05]$ in the second day as the propofol group made fewer correct choices than the control group; while there is no difference $[\mathrm{F}(1,18)=2.6, \mathrm{P}>0.05]$ in the third day (Figure 2).

But the propofol group's performance improved over the test days $[\mathrm{F}(2,27)=5.6, \mathrm{P}<0.05]$, whereas the control group performance stayed stable $[\mathrm{F}(2,27)=2.4, \mathrm{P}>0.05]$.

\subsection{Cross-Correlation Coherence between LFPs during Working Memory Test}

We measured the LFP signals 400 times from prefrontal cortex in 20 rats while they engaged in working memory task. Figure 3 shows raw data of the LFPs recorded from prefrontal cortex during a single test. Figures 3(a), (c) and (e) show raw data of LFPs recorded from one of the propofol rats in the first, second and third day respectively. Figure 3(b), (c) and (f) show raw data of LFPs recorded from one of the control rats in the first, second and third day respectively.

We calculated the average cross-correlation coherences of the LFPs across working memory task. Especially, we focused on cross-correlation coherences of LFPs around 2 s pre- and post-event, which was triggered by infrared in $\mathrm{Y}$-maze.

Figure 4 depicts the average cross-correlation coherences of the LFPs pre- and post- the working memory event. No significant differences in the average crosscorrelation coherences of the LFPs of propofol group were found between the pre and post the working memory event in the first $[\mathrm{F}(1,398)=1.5, \mathrm{P}>0.1]$ and second day $[\mathrm{F}(1,398)=1.9, \mathrm{P}>0.1]$. By contrast, the average cross-correlation coherences of propofol group increased significantly between the pre and post the working memory event in the third day $[\mathrm{F}(1,398)=8.1, \mathrm{P}<0.01]$.

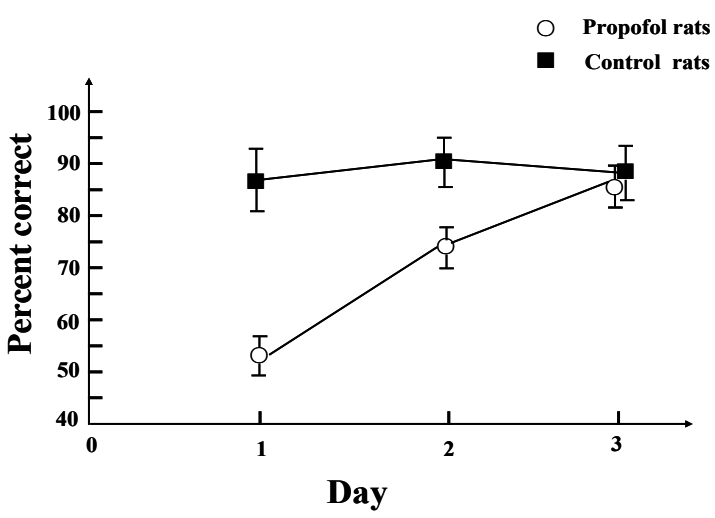

Figure 2. Reinforced alternation in the Y-maze. Mean correct choices rate $( \pm \mathrm{SE})$ during the test sessions where each test comprised a sample run and a choice run and an inter-trial interval of 2 min (continuous 10 tests in the morning and continuous 10 in the afternoon one rat one day). 


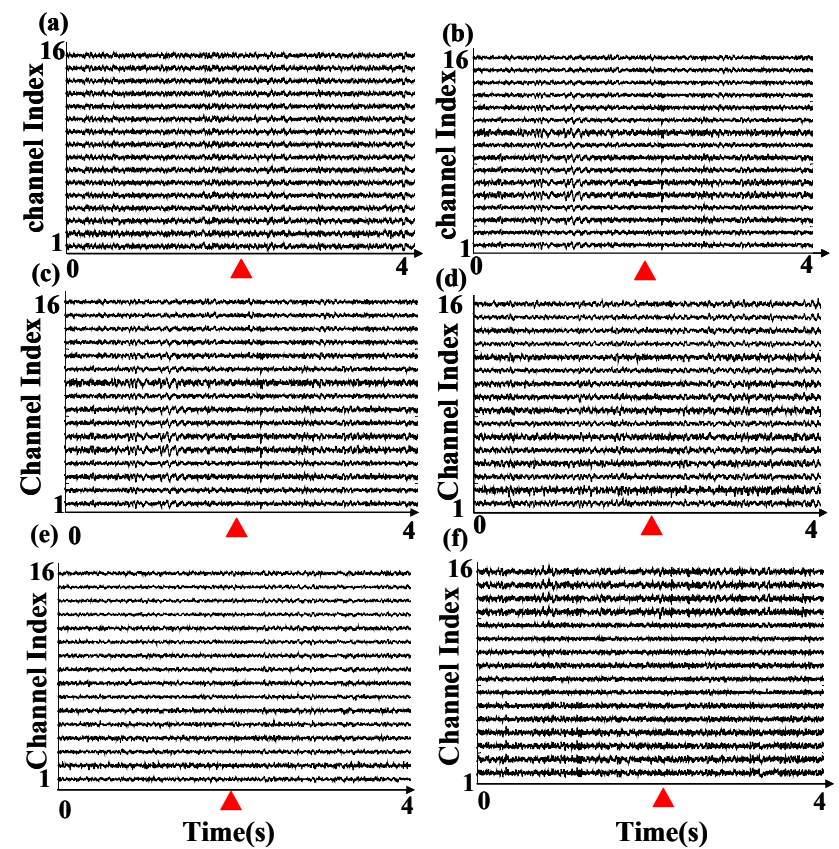

Figure 3. Example of LFP records. These data represent one test of each rat of two groups which contributed to the cross-correlation coherence estimates. $\boldsymbol{\Delta}$ represent the tripping time by infrared in Y-maze. (a), (c) and (e) represent one of propofol rat's LFP of first, second and third test day; (b), (d) and (f) represent one of control rat's LFP of first, second and third test day.

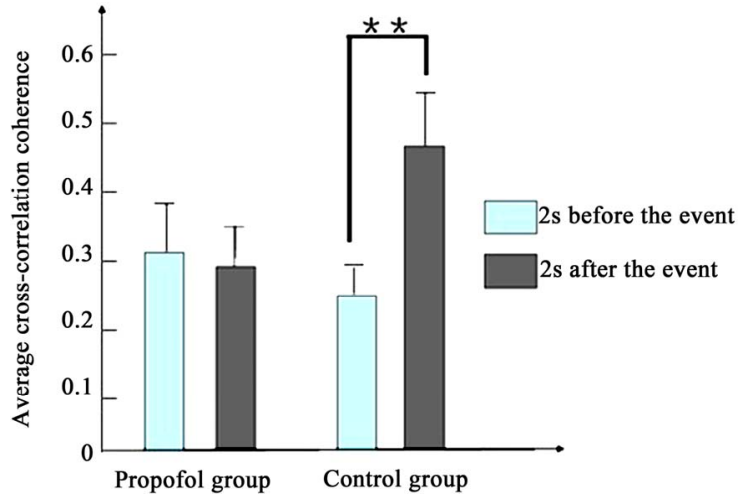

(a)

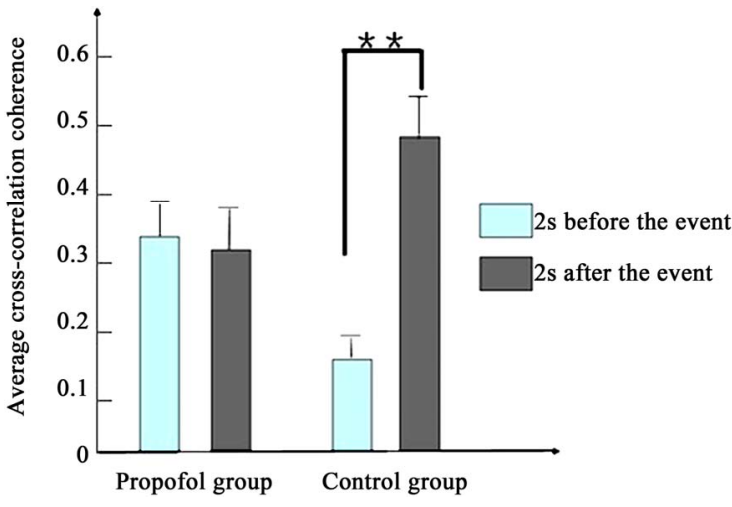

(b)

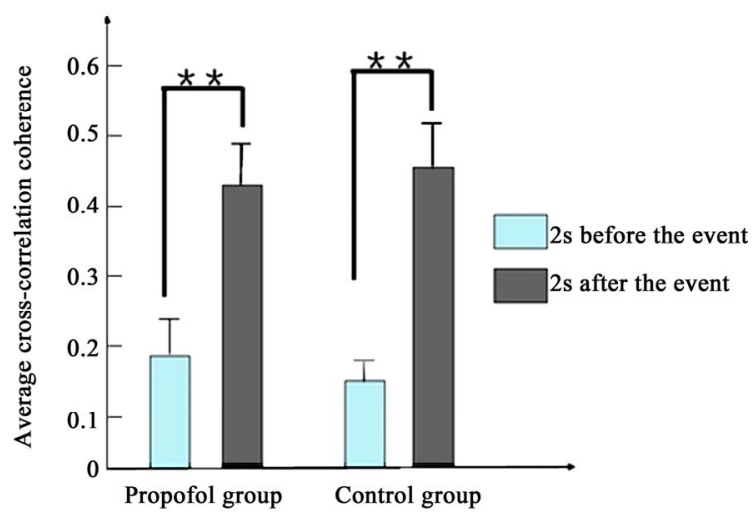

(c)

Figure 4. The average cross-correlation coherences of the LFPs of two groups pre and post the working memory event. (a) Presents the first day; (b) Presents the second day; (c) Presents the third day. ( ${ }^{*}$ present $\mathrm{P}<0.05,{ }^{* *}$ present $\mathrm{P}<0.01$ ). 
As to the control group, the average cross-correlation coherences increased significantly between the pre and post the working memory event every day $(\mathrm{P}<0.01)$.

The results indicate that propofol impairs the rats' working memory and cross-correlation coherence of LFPs in the prefrontal cortex. Our results suggest that Propofol can impair cross-correlation coherence of LFPs in the first two days, but not long time.

\section{DISCUSSION}

The purpose of this study was to evaluate the effects of propofol on working memory. From the result we can find out some common character: the propofol groups' correct choice rate is fewer than the control group in the first and second day; LFPs of propofol group can not form significantly synchronization in the first and second day either. In the third day there is no difference between the propofol and control group in behavior choice, and LFPs of propofol group rats can form significantly synchronization as the control group rats do. So we discover that whether LFPs of prefrontal cortex form significantly synchronization is relative to the rats' correct choice rate in the Y-maze, which can reflect function of rats' working memory. Propofol anesthesia can interfere with LFPs of rats' prefrontal cortex, and LFPs can not form meaningful synchronization when rats' working memory is formed in the Y-maze. But the effect of propofol on LFPs is not unrecoverable. On the third day, the effect of propofol on LFPs is disappeared, and LFPs can form significantly synchronization, then rats can form working memory again.

This research demonstrates that working memory is unimpaired in young rats when testing begins 3 days after general anesthesia with propofol. It is coincident with other studies which demonstrate that psychomotor and memory function are impaired for only $24 \mathrm{~h}$ or less after surgery under propofol anesthesia [27-29]. Propofol inhibits synaptic transmission in the central nervous system primarily by enhancing $\mathrm{GABA}_{\mathrm{A}}$ receptor currents while having smaller effects on NMDA receptor/channels. NMDA receptor blockade produces long-lasting changes in hippocampal LTP and LTD [30]. So propofol only partially inhibits LTP and has no effect of LTD [31]. LTP and LTD represent electrophysiological correlates of use-dependent efficacy of the synapse and are used to study mechanisms of memory formation. These evidences can also proof propofol anesthesia decreases working memory function during short time.

We use LFPs to reflect the effect of propofol on working memory for a number of reasons. First, the temporal structure of the LFP has been shown to reflect sensory and motor-related signals that can be modulated by cognitive processes. The LFP therefore provides additional information to single neuron activity [32]. Second, the source of LFPs is largely from synaptic activity, Thus, LFPs represent the inputs and local processing. Third, the cortical LFP is easy to record, even over long periods of time. Finally, compared with EEG, local field potential signals have better time and spatial resolution.

The degree of correlation between two LFPs can be calculated by use of mutual correlation function. There will be huge calculation if we calculate cross-correlation value of two LFPs among all 16 channel LFPs. So in this paper we select the LFP channel in which neurons with the highest discharge frequency exist during the working memory event as the reference channel, and calculate cross-correlation value between other 15 LFPs with reference LFP using method described in Toshiyuki, H. et al. [33]. Huge calculation can be saved by use of "reference" LFP.

However, the design of this study has important limitation. Because cognitive impairment is common immediately after surgery and general anesthesia in elderly patients, the three month old rats are as the youth for people, not the old ones. So in the future we will do more research about effect of propofol on working memory of aged rats.

In total, the result indicates that in young rats memory is not persistently impaired by propofol and that the anesthetic state is neither necessary nor sufficient for development of postanesthetic memory impairment.

\section{ACKNOWLEDGEMENTS}

This work was supported by two grants from the Natural Science Foundation of China (Grant No. 61074131 and Grant No. 91132722).

\section{REFERENCES}

[1] Jones, C., Griffiths, R.D. and Humphris, G. (2000) Disturbed memory and amnesia related to intensive care. Memory, 8, 79-94.

[2] Barr, G., Anderson, R.E., Owall, A. and Jakobsson, J.G. (2001) Being awake intermittently during propofol-induced hypnosis: A study of BIS, explicit and implicit memory. Acta Anaesthesiologica Scandinavica, 45, 834-838.

[3] Semba, K., Adachi, N. and Arai, T. (2005) Facilitation of serotonergic activity and amnesia in rats caused by intravenous anesthetics. Anesthesiology, 102, 616-623.

[4] Baeg, E.H., Kim, Y.B., Kim, H.T., Mook-Jung, I. and Jung, M.W. (2001) Fast spiking and regular spiking neural correlates of fear conditioning in the medial prefrontal cortex of the rat. Cerebral Cortex, 11, 441-451. doi:10.1093/cercor/11.5.441

[5] Lansner, A. (2009) Associative memory models: From the cell-assembly theory to biophysically detailed cortex simulations. Trends in Neurosciences, 32, 178-186. doi:10.1016/j.tins.2008.12.002

[6] Fuster, J.M. (1997) The prefrontal cortex. 3rd Edition, Raven Press, New York. 
[7] Funahashi, S., Bruce, C.J. and Goldman-Rakic, P.S. (1989) Mnemonic coding of visual space in the monkey's dorsolateral prefrontal cortex. Journal of Neurophysiology, 61, 331-349.

[8] Miller, E.K., Erickson, C.A. and Desimone, R. (1996) Neural mechanisms of visual working memory in prefrontal cortex of the macaque. Journal of Neuroscience, 16, 5154-5167.

[9] Funahashi, S. (2001) Neuronal mechanisms of executive control by the prefrontal cortex. Neuroscience Research, 39, 147-165. doi:10.1016/S0168-0102(00)00224-8

[10] Miller, E.K. and Cohen, J.D. (2001) An integrative theory of prefrontal cortex function. Annual Review of Neuroscience, 24, 167-202. doi:10.1146/annurev.neuro.24.1.167

[11] Wang, X.J. (2001) Synaptic reverberation underlying mnemonic persistent activity. Trends in Neurosciences, 24, 455-463. doi:10.1016/S0166-2236(00)01868-3

[12] Mitzdorf, U. (1987) Properties of the evoked potential generators: Current source-density analysis of visually evoked potentials in the cat cortex. International Journal of Neuroscience, 33, 33-59. doi: $10.3109 / 00207458708985928$

[13] Logothetis, N.K. (2003) The underpinnings of the BOLD functional magnetic resonance imaging signal. Journal of Neuroscience, 23, 3963-3971.

[14] Fries, P., Reynolds, J.H., Rorie, A.E. and Desimone, R. (2001) Modulation of oscillatory neuronal synchronization by selective visual attention. Science, 291, 1560-1563. doi:10.1126/science. 1055465

[15] Pesaran, B., Pezaris, J.S., Sahani, M., Mitra, P.P. and Andersen, R.A. (2002) Temporal structure in neuronal activity during working memory in macaque parietal cortex. Nature Neuroscience, 5, 805-811. doi:10.1038/nn890

[16] Siegel, M. and Konig, P. (2003) A functional gammaband defined by stimulus-dependent synchronization in area 18 of awake behaving cats. Journal of Neuroscience, 23, 4251-4260.

[17] Gail, A., Brinksmeyer, H.J. and Eckhorn, R. (2004) Perception-related modulations of local field potential power and coherence in primary visual cortex of awake monkey during binocular rivalry. Cerebral Cortex, 14, 300-313. doi:10.1093/cercor/bhg129

[18] Henrie, J.A. and Shapley, R. (2005) LFP power spectra in V1 cortex: The graded effect of stimulus contrast. Journal of Neurophysiology, 94, 479-490. doi:10.1152/jn.00919.2004

[19] Rickert, J., Oliveira, S.C., Vaadia, E., Aertsen, A., Rotter, S. and Mehring, C. (2005) Encoding of movement direction in different frequency ranges of motor cortical local field potentials. Journal of Neuroscience, 25, 8815-8824. doi:10.1523/JNEUROSCI.0816-05.2005

[20] Scherberger, H., Jarvis, M.R. and Andersen, R.A. (2005) Cortical local field potential encodes movement intentions in the posterior parietal cortex. Neuron, 46, 347-354. doi:10.1016/j.neuron.2005.03.004

[21] Taylor, K., Mandon, S., Freiwald, W.A. and Kreiter, A.K. (2005) Coherent oscillatory activity in monkey area v4 predicts successful allocation of attention. Cerebral Cortex, 15, 1424-1437. doi:10.1093/cercor/bhi023

[22] Womelsdorf, T., Fries, P., Mitra, P.P. and Desimone, R. (2006) Gamma-band synchronization in visual cortex predicts speed of change detection. Nature, 439, 733-736. doi: $10.1038 /$ nature 04258

[23] Nicolelis, M.A., Ghazanfar, A.A., Faggin, B.M., Votaw, S. and Oliveira, L.M. (1997) Reconstructing the engram: Simultaneous, multisite, many single neuron recordings. Neuron, 18, 529-537.

[24] De Coteau, W.E., Thorn, C., Gibson, D.J., Courtemanche, R., Mitra, P., Kubota, Y. and Graybiel, A.M. (2007) Oscillations of local field potentials in the rat dorsal striatum during spontaneous and instructed behaviors. Journal of Neurophysiology, 97, 3800-3805.

doi:10.1152/jn.00108.2007

[25] Courtemanche, R. and Lamarre, Y. (2005) Local field potential oscillations in primate cerebellar cortex: Synchronization with cerebral cortex during active and passive expectancy. Journal of Neurophysiology, 93, 20392052.

[26] Murthy, V.N. and Fetz, E.E. (1996) Oscillatory activity in sensorimotor cortex of awake monkeys: Synchronization of local field potentials and relation to behavior. Journal of Neurophysiology, 76, 3949-3967.

[27] Dressler, I., Fritzsche, T., Cortina, K., Pragst, F., Spies, C. and Rundshagen, I. (2007) Psychomotor dysfunction after remifentanil/propofol anaesthesia. European Journal of Anaesthesiology, 24, 347-354. doi:10.1017/S0265021506001530

[28] Kaoua, N.B., Veron, A.L., Lespinet, V.C., Claverie, B. and Sztark, F. (2002) Time course of cognitive recovery after propofol anaesthesia: A level of processing approach. Journal of Clinical and Experimental Neuropsychology, 24, 713-719.

[29] Sanou, J., Goodall, G., Capuron, L., Bourdalle-Badie, C. and Maurette, P. (1996) Cognitive sequelae of propofol anaesthesia. NeuroReport, 7, 1130-1132. doi:10.1097/00001756-199604260-00005

[30] Manahan-Vaughan, D., von Dorothea, H., Winter, C., Juckel, G. and Heinemann, U. (2008) A single application of MK801 causes symptoms of acute psychosis, deficits in spatial memory, and impairment of synaptic plasticity in rats. Hippocampus, 18, 125-134. doi:10.1002/hipo.20367

[31] Simon, W., Hapfelmeier, G., Kochs, E., Zieglgansberger, W. and Rammes, G. (2001) Isoflurane blocks synaptic plasticity in the mouse hippocampus. Anesthesiology, 94, 1058-1065. doi:10.1097/00000542-200106000-00021

[32] Mehring, C., Rickert, J., Vaadia, E., Cardosa, de Oliveira, S., Aertsen, A. and Rotter, S. (2003) Inference of hand movements from local field potentials in monkey motor cortex. Nature Neuroscience, 6, 1253-1254. doi: $10.1038 / \mathrm{nn} 1158$

[33] Toshiyuki, H. and Yasushi, M. (2005) Dynamically modulated spike correlation in monkey inferior temporal cortex depending on the feature configuration within a whole object. Journal of Neuroscience, 25, 10299-10307. doi:10.1523/JNEUROSCI.3036-05.2005 